\title{
O PLANEJAMENTO DE SUPRIMENTOS UTILIZANDO-SE A TEORIA DE FILAS E SIMULAÇÃO COMPUTACIONAL EM UMA OFICINA DE RECUPERAÇÃO DE COMPONENTES DE VAGÕES
}

Versão do autor aceita publicada online: 25 ago. 2021 Publicado online: 30 ago. 2021

Como citar esse artigo - American Psychological Association (APA): Felix, J. C., Peixoto, C. S., \& Edwige, C. A. (2021). O planejamento de suprimentos utilizando-se a teoria, de filas e simulação computacional em uma oficina de recuperação de componentes de vagões. Exacta. https://doi.org/10.5585/exactaep.2021.19577

\section{Josemar Coelho Felix}

felix.josemar@gmail.com

https://orcid.org/0000-0003-0220-2346

UFOP/Programa de Pós Graduação em Ciência da Computação

Tem experiência na área de Gestão da Manutenção, Engenharia Ferroviária, Informatica e educação. Atua principalmente nos seguintes temas: inclusão digital-educativa, popularização da ciência, educação empreendedora e inovação tecnológica. Além de ser membro do GETE(Grupo de Estudos em Tecnologia, Inovação e Empreendedorismo)

\section{Camila Silva Peixoto}

camilapeixoto94@hotmail.com

IFMG-Campus Congonhas/Engenheira de Produção

Técnica de Operação de Mina e Infraestrutura da Gerdau. Atuou como estagiária de Expedição e Assistente de Expedição na Empresa Cimentos Tupi.

\section{Camila Aparecida Edwiges}

camilaed20@gmail.com

IFMG-Campus Congonhas/Engenheira de Produção

Assistente de Tecnologia da Informação de Material Rodante-MRS Logística e Estudante de Engenharia de Produção no IFMG-Congonhas.

\section{RESUMO}

As empresas são mais competitivas, se elas tiverem um controle eficiente dos seus suprimentos. A aplicação da pesquisa operacional, com a utilização de ferramentas computacionais, pode ser uma alternativa de auxílio para evitar problemas no processo ferroviário. Esta pesquisa teve como objetivo, utilizar da mensuração dos parâmetros oriundos da Teoria de Filas e da simulação com o software Arena, além de ter um melhor diagnostico da previsão dos 
suprimentos na restauração de truques em uma oficina da MRS Logística. Com o estudo de caso aqui descrito, foi possível ter uma reflexão sobre a importância da simulação na produtividade, pois verificou que a variação do tempo de produção da manutenção, pode causar problemas no planejamento orçamentário do processo de manutenção dos truques.

Palavras-chave: Simulação Computacional. Planejamento de Suprimentos. Ferrovias.

\section{ABSTRACT}

Companies are more competitive if they have efficient control of their supplies. The application of operational research, using computational tools, can be an alternative aid to avoid problems in the railway process. This research aimed to use the measurement of parameters from Queue Theory and simulation with the Arena software, to have a better diagnosis of the supply forecast in the restoration of bogies in a MRS Logística workshop. With the case study described here, it was possible to reflect on the importance of simulation in productivity, as it was found that the variation in maintenance production time can cause problems in the budget planning of the bogie maintenance process.

Keywords: Computational Simulation. Supply Planning, Railways.

\section{INTRODUÇÃO}

O custo de um processo deve-se ser planejado adequadamente para alcançar o produto e ter o lucro desejado. Então o projeto de fabrica ideal é o local aonde as máquinas, os equipamentos, as pessoas e os suprimentos tem a devida sintonia com o planejamento orçamentário. Para constituir um produto, os elementos de informação devem estar em alinhados constantemente com o cliente e a busca por roteiros predefinidos, no qual a sequência de atividades necessita de determinados suprimentos, auxilia no controle da produtividade (Marujo, 2010).

Campos \& Belhot (1994) estudaram as dificuldades da gestão de manutenção de frotas de veículós e suas constatações levantaram que a qualificação da mão-de-obra, o nível de informalização, a complexidade do processo decisório e a necessidade de conferir a qualidade e a produtividade, se tornaram pontos chaves para trazer a ineficiência de um sistema de manutenção. Esses problemas impactam principalmente nos recursos humanos, materiais e consequentemente refletem negativamente nos custos. O que justifica a busca e o investimento em informações confiáveis e atualizadas da realidade da programação e planejamento das 


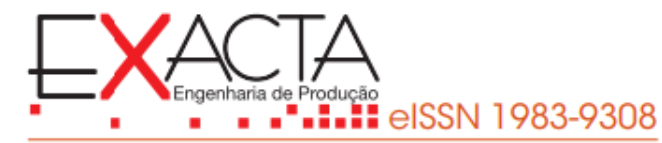

atividades e dos suprimentos, no qual a aplicação da pesquisa operacional com a utilização de ferramentas computacionais pode ser uma alternativa de auxílio. A simulação tem a capacidade de demostrar interatividade entre a ciência, o meio corporativo e a academia, ela garante maiores percepções de oportunidades e oferece benefícios para a iniciativa privada. Com a grande quantidade de dados gerados atualmente, existe necessidade de demostrar como a simulação consegue trazer industrias consideradas tradicionais a modernidade disponibilizada pela atual Indústria 4.0, a diminuição de erros nos processos produtivos assim como redução de custos (Marcelino et al.,, 2020).

As empresas são mais competitivas se elas tiverem um controle eficiente dos seus suprimentos e esses controles auxiliam no aumento da velocidade de operação e na confiabilidade das previsões de entregas. Diante desse cenário, analisar a capacidade produtiva dos ambientes, com ferramentas distintas, facilita a tomada de decisão e é um constituinte importante para a otimização de processo. Uma ferramenta que pode auxiliar nessa gestão de suprimentos é a teoria de filas, que pode ser conceituado como uma estrutura que detém um ou mais servidores que proveem serviços/atendimentos para certa quantidade de clientes que chegam a esses sistemas composto pelo processo de chegada, mecanismo de serviço e disciplina da fila (Alves et al.,2016).

O presente estudo teve como objeto de pesquisa uma empresa do setor ferroviário, localizada na cidade de Conselheiro Lafaiete/MG. Para a escolha da empresa, um dos fatores de decisão foi o fácil acesso aos dados sobre a produção e o fato de que o estudo realizado poderá ser aplicado na planta de produção, além de que as pesquisas nas empresas não são realizadas com simulações, o registro de hora/homem é pouco explorado e a empresa se propôs a criar modernização nos seus sistemas informacionais. A MRS Logística é uma operadora logística que administra uma malha ferroviária de $1.643 \mathrm{Km}$, nos estados de Minas Gerais, São Paulo e Rio de Janeiro. Atua no mercado de transporte ferroviário desde 1996, quando o governo transferiu à iniciativa privada a gestão do sistema ferroviário nacional. MBR, CSN, Congonhas Minérios, UPL, Vale, Gerdau e um grupo de pequenos investidores são os acionistas da empresa. Sua frota conta com 18 mil vagões e quase 800 locomotivas. Atualmente a empresa está entre as maiores ferrovias de carga do mundo, quase $20 \%$ de tudo o que o Brasil exporta e um terço de toda carga transportada por trens do país passam pelos trilhos da MRS. A empresa vive em constante evolução, desde a sua criação quase se quadriplicou o volume transportado, com operação em cinco portos. Possui indicadores de segurança e produtividade comparáveis as melhores ferrovias do mundo. 


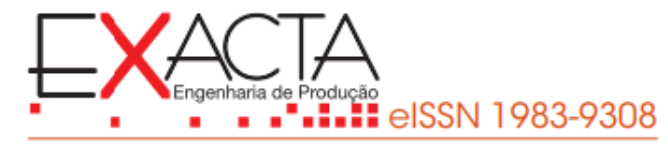

O foco das atividades da MRS está no transporte ferroviário de cargas gerais, como minério, produtos siderúrgicos acabados, cimento, bauxita, produtos agrícolas e contêineres. Para tanto, a empresa conta com a disponibilidade e confiabilidade de seus ativos, tornando-se essencial sua atividade de manutenção vinculada a suprimentos. Para Xenos (2014) a manutenção possui uma intensa relação com os setores produtivos, influenciando a qualidade e a produtividade. Desta forma, a manutenção possui um papel estratégico e de suma importância na melhoria dos resultados para a empresa, tanto operacionais quanto financeiros.

Neste sentido esta pesquisa tem como objetivo geral utilizar de conceitos da teoria das filas e simulação para criar um planejamento adequado da utilização de determinados suprimentos, para tentar evitar problemas no processo de restauração de truques ferroviários. Como objetivos específicos buscou-se analisar o desempenho da empresa no processo de recuperação de truques, através da mensuração dos parâmetros oriundos da Teoria de Filas e da simulação do processo utilizando o software Arena, tendo como objeto de estudo a previsão da utilização dos suprimentos chapa de desgaste da bolsa da cunha da travessa, Porca de Fixação do Transon e Parafuso Torx Cabeça Chata 1" para Truque. A partir dos resultados obtidos será realizado uma comparação com metas preestabelecidas pela empresa. A aplicação possibilitou ter uma reflexão sobre qual é a importância do planejamento e análise constante da produtividade, para prever o impacto em variáveis do processo, com a utilização da teoria de filas e a simulação computacional. Foi possível com aplicação destes dois métodos facilitar o entendimento mais detalhado da manutenção, o que consequentemente pode apontar compras desnecessárias de componentes, ou a parada do processo de recuperação pela falta de suprimentos.

\section{Referencial Teórico}

\subsection{Pesquisa Operacional}

Segundo Arenales (2015), a primeira conferência internacional de pesquisa operacional, foi realizada em Oxford na Inglaterra em 1952, no qual duas linhas de pesquisas foram evidenciadás, os de método matemáticos e problemas particulares de determinadas áreas. Se torna importante ressaltar por este mesmo autor, que apenas depois das décadas de 60, a área educacional e os setores públicos e privados começaram a colocar a área em evidência. A alta complexidade do processo, a dificuldade de alocar recursos, os conflitos internos de uma organização e os micro impérios dentro da organização, são pontos que fomentam o crescimento da Pesquisa Operacional. 


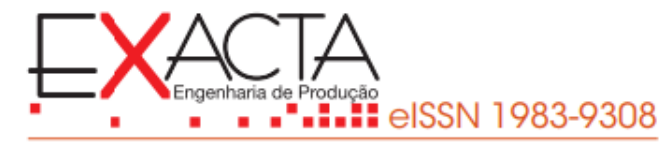

Quando há uma análise pelo ponto epistemológico, podemos determinar que a área em questão, se trata de ter a ação de buscar mais informações a respeito das operações. Para se realizar uma Pesquisa Operacional, deve-se buscar compreender o objeto de estudo, posteriormente abstrai a essência de problemas reais a partir da premissa que o modelo representa suficientemente a situação de representação e posteriormente são realizadas experimentações para validar a hipótese, para auxiliar o tomador de decisão (Hillier,2013).

Oque antes a Pesquisa operacional era utilizada apenas por militares, posteriormente a ciência administrativa utiliza desta técnica para auxiliar o mundo empresarial e acadêmico, na busca de soluções ótimas para problemas de processos e também para identificar pontos que precisam de intervenções. Os impactos da utilização desta área é a minimização dos impactos négativos que problemas e soluções na organização como um todo. Assim consegue-se reproduzir um funcionamento do sistema ou aumentar a produtividade, na busca por uma estrutura real e ideal de um processo (Melo \& Pessanha, 2011).

A Pesquisa Operacional é uma área que pode ser auxiliadora no dimensionamento do processo produtivo e na implementação de ideias. Projetar e viabilizar mudanças em um sistema produtivo, traz riscos e a cultura organizacional pode se contrapor a possibilidades de melhoria, por receio de consequências negativas e a obtenção de justificativas quantitativas, podem auxiliar na aceitação e até mudar opiniões. Quando as técnicas de modelagem e ferramentas de simulação são utilizadas, a área em questão pode conseguir considerar inter-relações e viabilizar abordagens científicas que são capazes otimizar sistemas, que possuem recursos escassos (Cauchick et al., 2011).

O Planejamento de Suprimentos determina as redes de fornecimento de material, a forma de registro da informação, suas fronteiras organizacionais, assim como o relacionamento entre a forma de agregar valor no suprimento até a geração de produto (Gaudreault et al., 2010). Quando se tem informações de um processo e conhecimento da Pesquisa Operacional, cria-se uma oportunidade de maximizar a satisfação dos clientes internos e externos de uma organização. Quando se tem domínio dessas duas áreas, cria-se uma melhor organização dos fluxos e processos dependentes de uma produção e na literatura se encontra informações de que Industrias Florestais (Sfeir, 2016), mineradora (Pimentel, 2011) e de Petróleo (Ribas et al., 2009), aumentaram a lucratividade graças a especialistas que dominaram esses dois temas simultaneamente. 


\subsection{Teoria de Filas}

Esperar em filas, faz parte de muitos lugares, como bancos, pedágios e supermercados. Apesar de ser necessárias as filas podem ser um sintoma mais visíveis de um sistema deficiente, mas também é inviável dimensionar um sistema para que seja extinta as filas. O objetivo é verificar se o processo está balanceado adequadamente, para que seja possível ter uma relação adequada do custo-benefício. A teoria das filas se é aplicável, quando à procura por uma determinada necessidade é superior à capacidade do sistema em atender a essa procura (Camelo, 2010).

A Teoria das Filas é um dos campos da Pesquisa Operacional, ela busca tratar problemas de congestionamentos de sistemas. Para se avaliar filas devemos avaliar o modelo de chegada dos usuários, modelo de serviço, número de atendentes, capacidade de atendimento e ordem de atendimento (Abensur, 2008).

Duarte (2008) citou Shamblin e Stevens Jr. (1987), para demostrar algumas definições importantes da teoria das filas, como: clientes é a unidade de chegada que necessita de ser atendido; fila é o número de clientes que espera o atendimento, mas só inclui o cliente que está na fila; o servidor é o processo ou sistema que realiza o atendimento do cliente, que necessariamente deve ser maior ou igual a 1 , sendo indicado pelo símbolo $\mathrm{k}$, o número de canais; taxa de chegada é a quantidade de clientes por período determinado de tempo, apartir do momento em que eles chegam; e tamanho da população é o tamanho do grupo que será clientes, quando é possível estimar a quantidade de clientes, se classifica como população é finita, caso contrário classifica-se como população infinita. Segundo estes mesmos autores as notações genéricas dos parâmetros da Teoria das Filas são:

$$
\begin{aligned}
& \lambda=\text { taxa média de chegadas } \\
& \frac{1}{\lambda}=\text { tempo médio ente chegadas de clientes sucessivos } \\
& \mu=\text { taxa média de atendimento } \\
& \frac{1}{\mu}=\text { tempo médio de atendimento de um cliente } \\
& k=\text { número de atendentes } \\
& n=\text { número de clientes no sistema }
\end{aligned}
$$




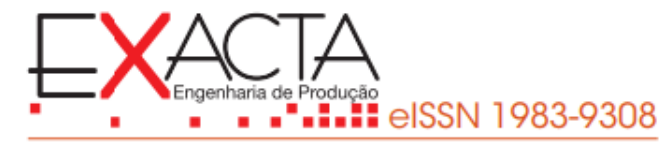

A taxa de utilização $(\rho)$ de um sistema de filas, pode demonstrar o quanto ele está utilizado e o quanto ele está ocioso. É possível calcular esse fator, com a utilização da Equação 1 sendo "S" e em algumas referências o "c", o número de atendentes disponíveis na fila.

$$
\rho=\frac{\lambda}{S \cdot \mu}
$$

Com essas informações consegue-se ter alguns parâmetros e compreender relações consideradas básicas para descrever e compreender um sistema. Torna-se importante para analisar os processos de fila, segundo Camelo (2010), utilizar a notação de Kendall, e levantar a distribuição dos intervalos entre chegadas, a distribuição do tempo de serviço, o número de canais de serviço ou capacidade de atendimento, o número máximo de clientes permitidos no sistema, o tamanho da população que fornece clientes e a disciplina da fila.

Em termos globais Andrade (2009) relata que um sistema de filas é composto por elementos que querem e/ou precisam ser atendidos e por um posto de serviço e que por ventura deve esperar até que este posto esteja livre. Em termos globais este mesmo autor, relata que um sistema de filas é composto por elementos que querem e/ou precisam ser atendidos e por um posto de serviço e que por ventura deve esperar até que este posto esteja livre.

\subsection{Simulação computacional e o uso do Software Arena}

Medeiros et al.(2015) discute a técnica de simulação e destaca o conjunto de saberes necessários para o seu domínio como a programação, otimização e programação dinâmica. Segundo este mesmo autor, a simulação pode ser considerada um processo de evolução natural dos seres vivos, por causa da possibilídade de simulação de modelos não reais com uma abordagem interdisciplinar, o que tornou a ferramenta importante para diversas áreas do conhecimento. A simulação está intimamente ligada a construção e conceito do que é um modelo, no qual um modelo é a interligação de várias variáveis de um sistema que determina o comportamento do conjunto.

O software Arena, pode ser um exemplo de sistema utilizado na simulação e ele apresenta um diferencial em sua tecnologia, pois as ferramentas de modelagem consistem em uma coleção de blocos que permitem registrar o desempenho do processo a ser analisado, de forma interativa e visual. A lógica do processo se baseia na ligação e configuração dos blocos (Silva et al., 2016). 
De acordo com Fernandes (2012) o Arena apresenta oito módulos básicos de fluxogramas, são eles:

- Create: Consiste nas entradas ou ponto de partida do modelo e identifica o tipo de entidade;

- Dispose: Se destina a saída ou ponto final do modelo de simulação;

- Process: Consiste no processamento das entidades, abrange os recursos disponíveis;

- Decide: Promove o desvio ao fluxo da simulação. Contém opções para que seja feita a tomada de decisões;

- Batch: Agrupamento de entidades, podendo ser temporárias ou permanentes. Quando temporárias podem ser desfeitas pelo módulo Separate;

- Separate: Separa as entidades que estão agrupadas;

- Assign: Associa figuras, atribui valores, entidades, entre outros;

- Record: Consiste na contagem de dados.

Também Fernandes (2012) apresenta além dos módulos de fluxogramas, sete blocos básicos para os módulos de dados, os quais são:

- Attribute: Permite definir o atributo certo para o modelo;

- Entity: Define tipos de entidades presentes;

- Queve: Permite alterar a ordem de prioridades em uma fila;

- Resource: Utilizado para definir os recursos disponíveis;

- Variable: Define a proporção de uma variável;

- Schedule: Quando utilizado junto com o módulo Resource, define um esquema de exploração de recursos;

Set: Consiste na definição do tipo de entidade, imagem e conjunto de recursos;

No Arena também encontramos a ferramenta Input Analyser, (Analisador de dados de entrada) a qual foi utilizada nesse estudo, para identificar a melhor curva estatística. Segundo Souza et al. (2015) o software tem sido uma ferramenta muito importante na simulação de processos. $\mathrm{O}$ Arena possui diversos recursos para realizar uma análise dinâmica do processo além de permitir visualizar tamanhos de filas, determinar gargalos, ocupação de recursos, verificar o comportamento do sistema, dentre outros, através de sua interface gráfica, planilhas auxiliares, relatórios e apresentações de animações. Integrado a ferramenta possui também recursos 3D. 


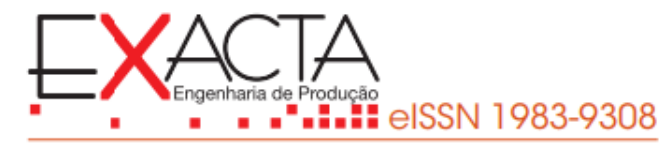

A representação do tráfego de trens em estudos de simulação é uma tarefa complexa, que exige conhecimentos específicos das operações ferroviárias. O Arena pode auxiliar nos, diversos conflitos que ocorrem diariamente pelos centros de controle tráfego. Pedreira Junior e Nascimento (2015) utilizou a simulação pelo programa citado para construir um modelo adequado e compreender problemas no transporte de minério de ferro na Ferrovia de Integração Oeste Leste (FIOL), que está sendo construída nos estados da Bahia e do Tocantins. Posteriormente a qualidade do modelo foi testada e constatou-se a eficiência da utilização quando conseguiu-se comparar os parâmetros operacionais planejados do projeto, com as saídas geradas pelo modelo desenvolvido.

Existem outros estudos sobre a utilização do Arena no transporte ferroviário de carga, pois as decisões de logística, impactam cada vez mais nos negócios das empresas nos quesitos relacionados à competitividade. Dentro desse contexto, a pesquisa de Faria (2016), utilizou a ferramenta computacional para auxiliar na validação de análises das três esferas de planejamento. Vale destacar que suas pesquisas no nível operacional, incentivou mudanças nas estratégias de manutenção programada de via permanente, pois elas impactam consideravelmente a capacidade da operação de trem, sendo bem aderentes ao sistema real com $95 \%$ de confiança.

Mas dentro da literatura é possível encontrar aplicação distintas do Arena como a análise do sistema de estocagem de produtos, pois o software pode ser utilizado para alcançar o objetivo de melhorar a produtividade do sistema e o nível dos serviços oferecidos aos clientes. O sistema atual de estocagem dos produtos, nas prateleiras, é feito de forma aleatória. Em conjunto com outras ferramentas relacionadas a custos, constatou-se com a simulação que a minimização das distâncias para identificar um novo modelo de organização, dos produtos de maior rotatividade e nas prateleiras, pode ser uma alternativa para melhorar o processo produtivo de indústrias como a moveleira. A constatação que foi levantada, partiu-se de uma comparação entre as performances dos sistemas atual e proposto dos estudos de Santos \& Grander(2016).

\section{Metodologia}

O foco deste trabalho está na disponibilidade de um suprimento que ocorre na manutenção de uma das oficinas de vagões da empresa. O congestionamento de ativos, neste caso os truques de vagões, é um problema significativo que a empresa enfrenta. Surge então, uma dificuldade de relacionar demanda dos suprimentos detalhados abaixo, com os ativos que necessitam deste material para a manutenção e os valores. 
- Chapa de desgaste da bolsa da cunha da travessa: Valor unitário de $\mathrm{R} \$ 16,15 /$ Quantidade em unidade:800

- Porca de Fixação do Transon: Valor unitário de R\$ 38,51/ Quantidade em unidade:200

- Parafuso Torx Cabeça Chata 1" para Truque: Valor unitário de R \$ 9,70/ Quantidade em unidade:500

Esta pesquisa pode ser caracterizada como um estudo de caso, de natureza empírica, pois investiga um fenômeno contemporâneo, dentro de um contexto real e as fronteiras não estão claramente definidas, porque talvez para o entendimento da sistemática seja necessário o aprofundamento de outras variáveis. O estudo justapôs dados obtidos em campo na área operacional da empresa, aos conhecimentos técnicos acadêmicos com a aplicação de técnicas da Teoria das Filas e a seguir são detalhadas as etapas e atividades que foram utilizadas para a coleta dos dados.

Etapa 1 - Realizou-se a ambientação do local identificando e verificando os principais pontos de que deve ser levado em consideração, definir os principais recursos tanto físicos quanto humanos. Então se vê necessário entender que o objetivo do ambiente intitulado Oficina de Recuperação de Truques, busca garantir a confiabilidade dos vagões da MRS Logística. Esse componente do vagão é constituído por várias peças e busca uma melhor distribuição da lotação do vagão, a flexibilidade e acomodação, para evitar o atrito entre as peças, trazer a estabilidade, evitar o descarrilamento, garantir o sistema mecânico de suspenção, amortecer as vibrações e garantir da melhor forma a eficiência do sistema de freio.

Etapa 2 - Estudaram-se os procedimentos de maneira a compreender o passo-a-passo da meta do local, então a recuperação de truques, pode ser explicada, de forma superficial em três etapas a desmontagem, realizada por dois mantenedores mecânicos, a soldagem, realizada por um mantenedor metalúrgico, e a montagem, realizada pelos mesmos dois mantenedores mecânicos que realizam a desmontagem. Para a aplicação da teoria das Filas e a aplicação de simulação computacional, a fase que se estudou fora a recuperação parcial da travessa central, componente do truque responsável por transmitir o peso do vagão para os truques (sistema de rodas ligadas por um eixo) e os Parafuso Torx Cabeça Chata 1" e Porca de Fixação do Transon da travessa lateral, peça inteiriça do truque, que é possível visualizar na Figura 1.

Etapa 3 - Recolheu em um sistema de informações amostras sobre o tempo gasto por cada mantenedor no processo de manutenção de truques. Esses dados foram utilizados no software 
Arena e comparado com o método de cronoanálise e as equações da Teoria de Filas, descritas na revisão da literatura deste artigo.

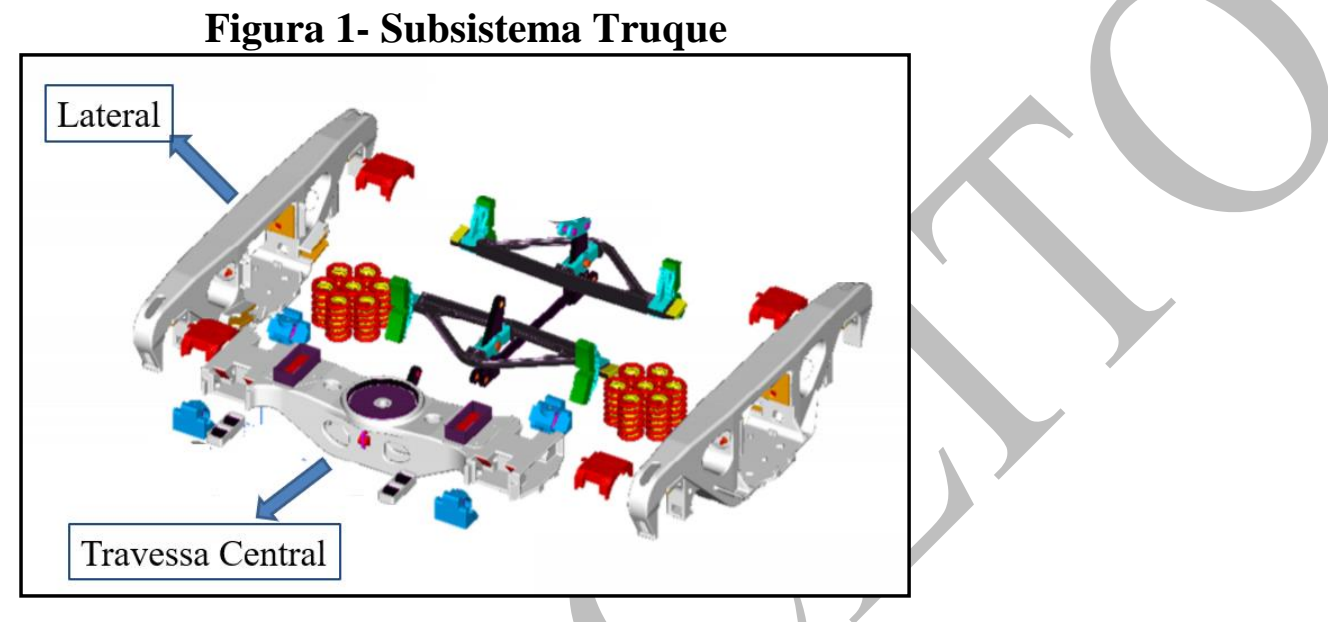

Fonte: adaptado de Fundamentos de vagões MRS, 2015.

As más condições destes suprimentos podem comprometer a confiabilidade do subsistema anteriormente contextualizado, o que justifica a buscar por informações detalhadas sobre a compra e a utilização e assim trazer uma perspectiva de planejamento, e garantir a eficácia da tarefa de manutenção. Então tornou-se necessário na Figura 2, a construção do mapa fluxograma e Activity Cicle Diagram (ACD), para demostrar a movimentação entre os processos de desmontagem, recuperação e montagem dentro do layout da área de recuperação de truque, para facilitar a visualização do processo. 
Figura 2- Mapa fluxograma do processo de recuperação de truque e modelo ACD.
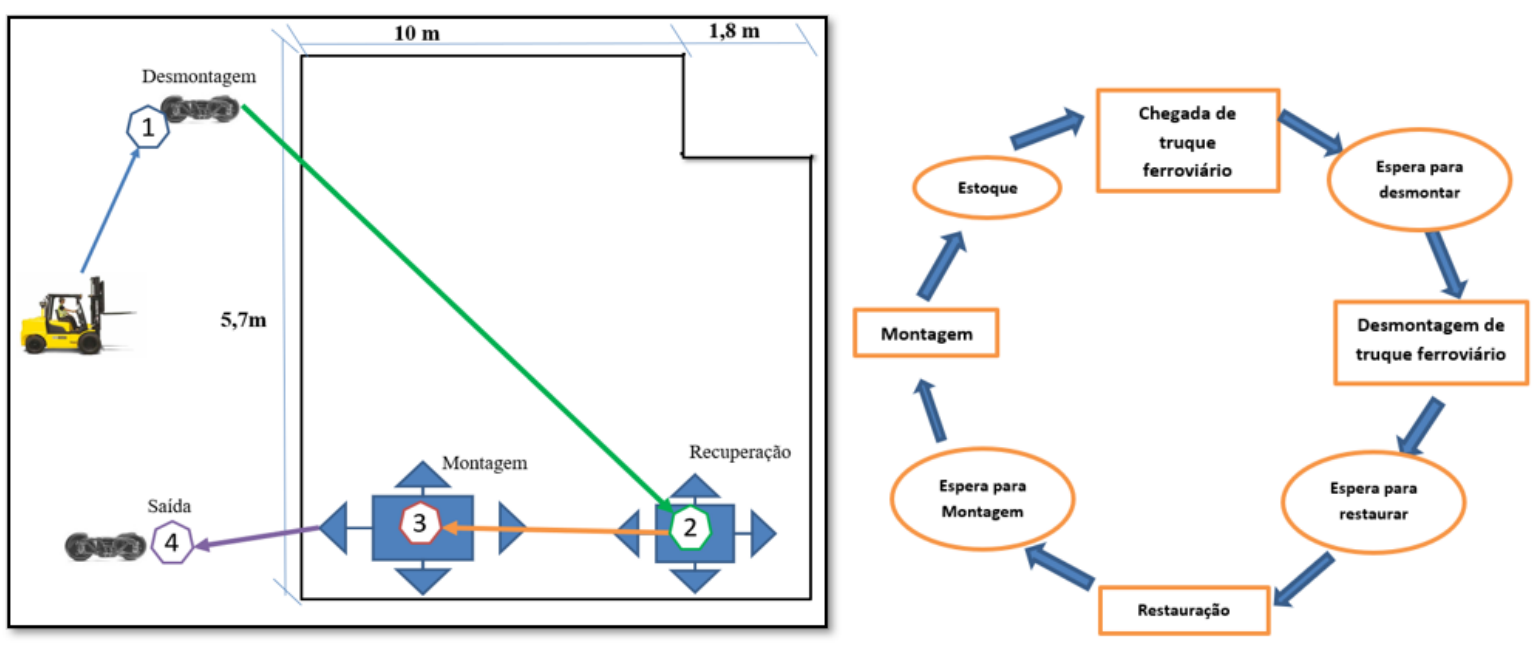

Fonte: MRS Logística.

Diante da observação do ambiente em questão a ser estudado, em que têm-se 3 servidores (no caso mantenedores que trabalham na recuperação), foi aplicado o modelo M/M/3 de sistema de filas, após a construção do modelo conceitual Activity Cycle Diagrams(ACD) possível de se visualizar na Figura 2.

Segundo Prado (2006), o modelo M/M/1 mencionado se caracteriza como um sistema com um único atendente em que tanto as chegadas (definidas pelo parâmetro $\lambda$ ) quanto o atendimento (definido pelo parấmetro $\mu$ ) são Markovianos, ou seja, seguem as distribuições de Poisson ou exponencial negativa, sendo que nesta pesquisa a população é infinita. Os valores de $\lambda$ e $\mu$ são o ritmo médio de chegada de usuários por unidade de tempo e ritmo médio de atendimento de cada atendente por unidade de tempo, respectivamente. Na Tabela 1 se encontra as variáveis que serão analisadas durante a pesquisa, com suas respectivas equações. 
Tabela 1: Variáveis do modelo M/M/1 que serão estudadas durante a pesquisa.

\begin{tabular}{ccc}
\hline $\begin{array}{c}\text { Nome da } \\
\text { variável }\end{array}$ & Descrição & Fórmula \\
\hline NF & Número médio de clientes na fila & $N F=\frac{\lambda^{2}}{\mu(\mu-\lambda)}$ \\
NS & Número médio de clientes no sistema & $N S=\frac{\lambda}{\mu-\lambda}$ \\
TF & Tempo médio durante o qual o cliente fica na fila & $T F=\frac{\lambda}{\mu(\mu-\lambda)}$ \\
TS & Tempo médio durante o qual o cliente fica no & $T S=\frac{1}{\mu-\lambda}$ \\
$\rho$ & sistema & $\rho=\frac{\lambda}{\mu}$ \\
$P_{0}$ & Probabilidade de não existir ninguém no sistema & $P_{0}=1-\frac{\lambda}{\mu}$ \\
\hline
\end{tabular}

Fonte: Prado (2009).

Depois de aplicado o modelo M/M/1 de sistemas de filas, foi implementado o modelo computacional utilizando o software Arena, que é uma poderosa ferramenta para realizar simulações e análise de cenários. Por fim, está pesquisa se classifica como quantitativa pois obtém números para criar discussões e auxiliar no desenvolvimento de melhorias, através de modelagem e simulação. Também por causa dos procedimentos técnicos aqui abordados, é possível declarar que esse estudo se classifica como um estudo de caso, pois aumenta o conhecimento de um objeto a partir da coleta direta de dados, pois foi necessária para levantar dados para obter cronoanálise e mineração de dados, porque utilizou-se de um sistema computacional responsável por cronometrar as atividades (Prodanov \& Freitas, 2013).

\section{Resultados e Discussões}

\subsection{Resultados da aplicação da Teoria das Filas}

Para o levantamento dos dados sobre o ritmo de atendimento, utilizou-se a ferramenta de tempos e movimentos, intitulada cronoanálise(Tabela 2), sendo que através dela buscou-se analisar o tempo necessário para a realização das 3 tarefas, que garantem a manutenção parcial do truque ferroviário. O tempo de atendimento é a soma dos 3 processos analisados na tabela informada sendo possível realizar a recuperação em 1 hora e 50 minutos. Porém, como regra 


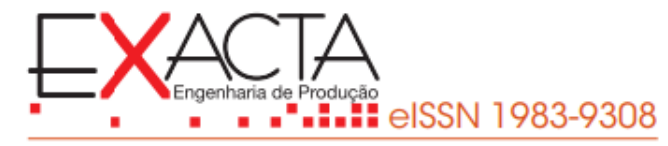

Neste estudo o processo de troca de parafusos e porcas acontecem na desmontagem do truque e para garantir que os dois suprimentos irão acabar juntos, então foi colocado como premissa deste estudo que há 5 lotes de porcas e 2 lotes de parafusos no ano, o que garante o total de 1000 unidades para os dois suprimentos, mas como é utilizado 3 unidades de porca e de parafuso por vez considera-se 166 conjuntos. Considerando que o total de dias úteis nesse intervalo foi de 66 dias têm-se que (166 conjuntos/66 dias) = 2,53 conjuntos/dia.

Para mensurar o ritmo de atendimento $(\mu)$ considerou-se o tempo de atendimento (TA) de cada chapa, obtido através da cronoanálise e apresentado na Tabela 3 e acrescentando a tolerância de $18,4 \%$ em cada processo. Logo, quando se considera o valor de TA igual a 14 min/chapa e $171 \mathrm{~min} /$ atendimentos por conjunto de porcas e parafusos, e considerando a equação $\mu=\frac{1}{T A}$, constatou-se que a taxa de atendimento é de 4,17 atendimentos/hora para a chapa e 0,35 atendimentos/hora para os conjuntos de porcas e parafusos. A empresa tem uma política de que, do total da jornada diária, o funcionário passa $52 \%$ desse tempo efetivamente executando atividades direta a manutenção de componentes do vagão (no caso essa porcentagem totaliza 4,16 horas) e o restante é despendido com treinamentos de segurança. Então considerando essas informações temos a taxa de atendimento $(\mu)$ igual a 17,8 atendimentos/dia para as chapas e 1,45 atendimento/dia para o conjunto porca/parafuso. Percebe-se que a capacidade de atendimento $(\mu)$ é maior que o ritmo de chegada $(\lambda)$, então constata-se que o sistema pode ser caracterizado como estável, sem a existência de filas infinitas. De posse dos valores das taxas médias diárias pode-se aplicar as fórmulas do modelo M/M/1 e estimar variáveis definidas para análise das filas, conforme mostrado abaixo:

- Taxa média diária de chegadas ( $\lambda$ ): com o valor para a chapa de 12,12 e valor para o conjunto de porca e parafuso de 2,53(Unidade considerada peças ou conjuntos/dia).

- Taxa média diária de atendimento $(\mu)$ : com o valor para a chapa de 17,8 e valor para o conjunto de porca e parafuso de 1,45(Unidade considerada Peças/dia).

- Número de clientes em fila (NF): com o valor para a chapa de 1,45 e valor para o conjunto de porca e parafuso de 6,18(Unidade considerada Peças em fila).

- Número de clientes no sistema (NS): com o valor para a chapa de 2,13 e valor para o conjunto de porca e parafuso de 7,05 (Unidade considerada Peças no sistema).

- Tempo médio em fila (TF): com o valor para a chapa de 0,119 e valor para o conjunto de porca e parafuso de 4,86 Horas. 
- Tempo médio no sistema (TS): com o valor para a chapa de 0,176 e valor para o conjunto de porca e parafuso de 5,55 Horas.

- Taxa de utilização $(\rho)$ : com o valor para a chapa de $68 \%$ e valor para o conjunto de porca e parafuso de $88 \%$.

- Probabilidade de não haver ninguém no sistema $\left(P_{0}\right)$ : com o valor para a chapa de $32 \%$ e valor para o conjunto de porca e parafuso de $12 \%$.

\subsection{Resultados da aplicação da aplicação da simulação no software Arena}

Após a delimitação do processo a ser estudado, foi realizado a coleta de dados acerca dos intervalos de entrada dos truques no sistema e dos tempos de atendimento nas etapas de desmontagem, recuperação e montagem dos truques na oficina de manutenção.

Com relação as entradas, observou-se que os truques chegam a uma taxa constante de 4 truques por dia, ou seja, aproximadamente 0,17 truques por hora. Utilizando a equação da Teoria de Filas, IC $=1 / \mu$, transformou-se a taxa em intervalos entre chegadas, obtendo-se o valor de IC = 6 horas. Isso foi feito devido ao fato de que o software de simulação Arena que será utilizado para execução deste trabalho somente aceita dados de intervalos entre chegadas.

Com relação ao atendimento, os tempos foram coletados a partir da base de dados da própria empresa em questão, sendo levantado 95 amostras para cada um dos processos, conforme apresentado no apêndice $A$.

De posse desses dados, foi necessário definir as expressões que mais se aproximam da distribuição de probabilidade dos tempos de atendimento de cada processo. Para tal aplicou-se a ferramenta Input Analyser presente no programa Arena. Sendo assim, alimentou-se a ferramenta com os dados numéricos e na Tabela 3 é demostrado a distribuição de probabilidade e suas expressões matemáticas que serão utilizadas no modelo lógico.

Tabela 3 - Descrição das expressões matemáticas e valores estatísticos encontrados.

\begin{tabular}{cccc}
\hline Etapas & Distribuição & Expressão (em horas) & Erro Quadrático \\
\hline Desmontagem & Beta & $1.75 *$ BETA $(2.59,2.88)$ & 0.001708 \\
Recuperação & Triangular & TRIA $(0,0.749,2.41)$ & 0.002345 \\
Montagem & Norma & NORM $(1.62,0.453)$ & 0.003008 \\
\hline
\end{tabular}

Fonte: Elaborado pelos autores. 
Durante o levantamento de dados também foi observado que existem 3 funcionários, na função de mantenedor, para a realização das atividades de manutenção do truque, sendo que esses funcionários trabalham numa jornada diária de 8 horas, com parada de 1 hora para almoço. Além disso, diariamente estes mantenedores precisam passar por interrupções no serviço para treinamentos de segurança, sendo a duração destas paradas listadas abaixo:

- Análise Preliminar de Risco: 0,84 horas, para 60 pessoas e é feito diariamente;

- Pré uso: 0,667 horas para 60 pessoas e é feito diariamente;

- Diálogo Diário de Segurança: 0,32 horas para 60 pessoas e é feito diariamente;

\subsubsection{Modelagem Computacional}

A pesquisa objetiva elaborar um planejamento de compras para três suprimentos específicos que são empregados no sistema da empresa estudada logo, para alcançar este objetivo, foi elaborado um modelo que simula o processo de manutenção do truque, com suas respectivas etapas, e com os resultados obtidos na simulação, estipulou-se as necessidades de compras para estes suprimentos.

Para a construção do modelo computacional recorreu-se ao modelo conceitual e fluxograma do processo, apresentados anteriormente na metodologia deste artigo. $\mathrm{O}$ template do modelo e as especificações de cada módulo usado na modelagem estão apresentados na Figura 3. 
Figura 3 - Template do modelo computacional da oficina de truques.

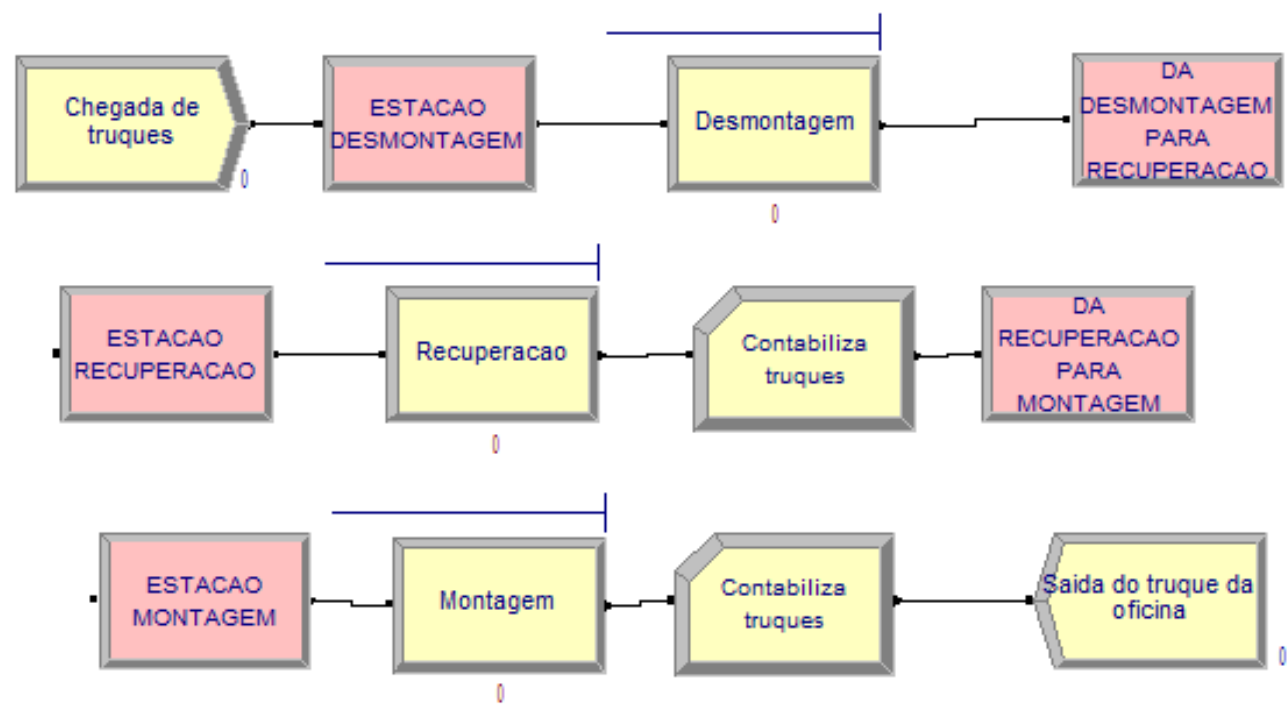

Fonte: Elaborado pelos autores.

A entidade considerada no fluxograma foi o truque, e sua chegada ao sistema está representada no módulo Create do modelo. Os processos de desmontagem, recuperação e montagem foram dispostos no módulo Process, sendo que os mantenedores são os recursos. Para a execução de suas atividades os mantenedores precisam se deslocar entre os três processos, gastando um tempo de 6 minutos, por isso estes foram divididos em três estações, utilizando os módulos Station e Leave.

Conforme descrito anteriormente, o sistema sofre interrupções diárias devido paradas com almoço dos funcionários e atividades voltadas à segurança. Estas interrupções foram adicionadas aos recursos do modelo (no caso, os mantenedores), através do módulo Schedule, para que os resultados obtidos sejam mais condizentes com a realidade da oficina. Também foi inserido o módulo Record depois dos processos de recuperação e montagem, para contabilizar a quantidade de vezes que os truques entram nesses processos, possibilitando a mensuração da quantidade de chapas, porcas e parafusos que seriam necessárias em um dia de serviço, visto que é nesses dois processos que estes suprimentos são utilizados. Com isso, consequentemente será possível a elaboração de um planejamento de demanda desses suprimentos e dos seus respectivos gastos.

O modelo foi replicado por 3 meses (66 dias úteis), sendo a duração da replicação de 8 horas, considerando 24 horas por dia. A base de tempo adotada no modelo e apresentada nos relatórios é em horas. 


\subsection{Discussão dos Resultados}

Através da análise dos relatórios gerados pelo Arena, obtido com a simulação, verificou se como determinados recursos se comportam diante do cenário real e constatou-se que, com o quadro atual, dos 4 truques que chegam ao sistema, em média 3 concluem o processo, o que é um indicador de que o processo está operando com eficiência. Observou-se também que o tempo médio de execução da manutenção dos truques foi de 5,8796 horas.

Com relação ao relatório sobre filas (Quеиes), analisou-se os parâmetros de tempo médio na fila (Waiting Time) e tamanho médio da fila (Number Waiting) e percebeu-se que a etapa de desmontagem teve maior tempo médio de fila (1,9752 horas), visto que, em algum momento da simulação, houve um tempo máximo de espera de 3,066 horas, assim como em um certo momento esse tempo de espera chegou a $0 \mathrm{~h}$, demonstrados nas colunas Maximum Value e Minimum Value respectivamente. Possivelmente isso pode ter ocorrido devido à falta de algum suprimento no estoque, a complexidade da atividade ou algum outro acontecimento que não estava previsto.

O tamanho médio de fila da desmontagem também foi o maior, com aproximadamente 1 truque em fila, e constatou-se pelo Maximum Value que, em determinado momento os 4 truques que entraram no sistema ficaram aguardando atendimento, podendo esse gargalo ser justificado pelos mesmos motivos explicados anteriormente no tempo de fila.

Com relação ao relatório sobre os recursos (Resource), foi possível analisar informações específicas aos recursos do sistema, que representam recursos humanos, no caso os mantenedores, dispostos no sistema, e observou-se que a taxa de utilização individual (Instantaneous Utilization) do mantenedor foi de aproximadamente 60\%, e que a taxa de ocupação dos recursos de forma conjunta (Number Busy) foi de 1,58 ou (158\%). O percentual de ocupação em relação ao tempo disponível (Scheduled Utilization) foi de 1,1235 ou 112\%. Além disso, o número de vezes que utilizou-se o recurso (Total Number Seized) foi aproximadamente 12 vezes.

O relatório também demonstrou a quantidade de vezes que a entidade (truques) passou pelos processos de recuperação e montagem, conforme Figura 4. Percebe-se que, em média, saíram 4 truques do processo de recuperação, sendo que o número mínimo de entidades que chegaram a sair dessa etapa foi de 3 truques. Com relação ao processo posterior de montagem, o número médio de entidades que saíram foi de 3 truques, sendo que em determinado momento chegaram 
a sair apenas 1 truque dessa etapa. Isso pode ter ocorrido devido à falta de suprimentos em estoques, e ressalta-se que os suprimentos que são empregados nesses dois processos são as chapas, os parafusos e porcas, fato este que reforça a importância deste estudo para o planejamento da demanda e compras destes insumos.

\section{Figura 4 - Relatório para contabilizar a quantidade de truques que saem dos processos.}

\section{Counter}

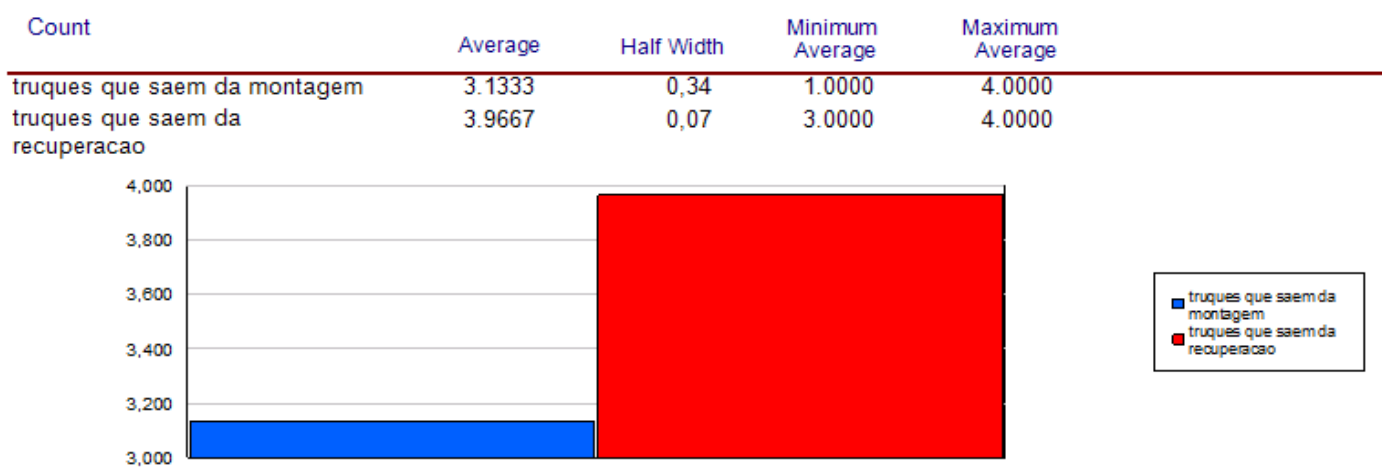

Fonte: Elaborado pelos autores.

Com a análise da teoria de filas, associada a cronoanálise foi possível obter o total de recuperação de 67 truques recuperados, logo o sistema consumiria 268 chapas, 201 parafusos e 201 porcas, o que resultaria em gastos de $\mathrm{R} \$ 4329,00 ; \mathrm{R} \$ 7740,51$ e $\mathrm{R} \$ 1949,70$ respectivamente, totalizando em $\mathrm{R} \$ 14019,21$ gastos no período de três meses. Em contrapartida, com a simulação obteve-se uma média de 3 truques atendidos em um dia, o que resulta em aproximadamente 198 truques recuperados considerando o mesmo intervalo de tempo de 66 dias, logo o sistema consumiria 792 chapas, 594 parafusos e 594 porcas, o que resultaria em gastos de $\mathrm{R} \$ 12790,80 ; \mathrm{R} \$ 22874,94$ e $\mathrm{R} \$ 5761,80$ respectivamente, totalizando um gasto de R $\$ 41427,54$ em três meses. Então constatamos que seria dada uma diferença no orçamento de $\mathrm{R} \$ 24408,33$ no período de 3 meses.

Também foi encontrado discrepâncias na taxa de ocupação, que na Teoria de filas foi de $68 \%$, ao passo que com a modelagem esse valor foi de $60 \%$ para um mantenedor. A ociosidade dos recursos nas duas aplicações pode ser justificada, devido o tempo que o mantenedor gasta com outras atividades, como por exemplo, a preparação do ambiente, vestir as roupas próprias para o serviço. 


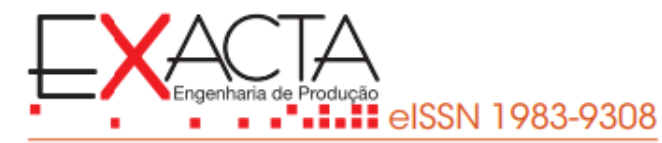

Atualmente a empresa baseia-se nos dados da cronoanálise para realizar seu planejamento de compras, porém ao realizar o levantamento dos dados em campo percebe-se que este é menor, a ao analisar os resultados constate-se que os funcionários realizando suas atividades de forma mais rápida que o preestabelecido, e consequentemente a empresa tem gastado mais suprimentos do que o previsto, o que implica muitas vezes em paradas no processo devido à falta desses insumos em estoque. Além disso, a empresa estabelece uma meta de que por dia sejam recuperados três truques, concluindo-se que somente os resultados da simulação atendem ao que foi preestabelecido.

Recomenda-se fazer comparação constante na evolução e na rapidez do desenvolvimento da manutenção, porque em muitos momentos a busca pelo menor tempo, pode trazer impactos negativos, não só na busca pelo alinhamento orçamentário, mas também na rotatividade de funcionários e na segurança do processo. Logo como melhoria para a empresa sugere-se que esta realize seu planejamento de demanda de suprimentos de acordo com os tempos recolhidos em campo e com o que foi alcançado com a modelagem computacional, visto que estes são mais adequados a realidade da empresa e mostra que o ser humano não atua de maneira linear.

Nos dias atuais a empresa tem uma forte preocupação com a redução de custos e estuda seus processos, condições de trabalho e destinação de insumos. Avaliações que trazem discrepâncias na preocupação orçamentária, atualmente está sendo um dos focos dos gestores. Verificar qual desses métodos está mais alinhádo ao processo atual, é um motivo para aumentar os investimentos de pesquisas científicas no setor ferroviário (Felix et al., 2021).

\section{Conclusões}

A aplicação da teoria de fillas na análise da utilização dos suprimentos e o seu reabastecimento, auxilia no processo de decisão para compras e identificação da capacidade de melhoria do processo. Torna-se possível evitar gastos não planejados e a falta de material, prejudicando a produção e manutenção de determinados componentes dos vagões. No presente artigo, buscouse apresentar os conceitos de Teoria das Filas e de Simulação computacional, bem como a possibilidade de uso no diagnóstico do desempenho de uma empresa no setor de manutenção de vagões, para facilitar o gerenciamento dos suprimentos. Como contribuições para a teoria, no referencial teórico foi descrito e apontado pesquisas que utilizam de tais técnicas, para realizar melhorias em ambientes industriais.

Por meio das fórmulas matemáticas do modelo M/M/1 e simulação computacional do processo de manutenção dos truques foi possível estimar a quantidade média de truques e o gasto 


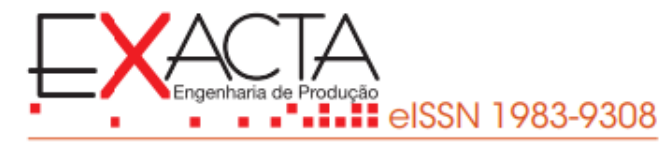

trimestral máximo com os suprimentos estudados. No entanto, comparando os resultados entre os dois métodos, percebe-se que estes foram bem discrepantes com relação a produtividade e consequentemente com os gastos da empresa com suprimentos. Contatou-se então que não é possível fazer uma previsão somente com a aplicação da Teoria das filas e cronoanálise, visto que as amostras não são uniformes e contínuas e não se adequam corretamente às reais necessidades da empresa.

A simulações realizadas proporcionaram uma reflexão sobre a forma de se realizar planejamento orçamentário na produção da manutenção da MRS Logística. A taxa de ocupação do mantenedor é variável de acordo com cada realidade da equipe, pois se houver grande absenteísmo e problemas com planejamento de férias, a busca pela ocupação do mantenedor é maior. Se o mantenedor conseguir manter o ritmo de trabalho anterior, suâ produção irá aumentar e a utilização de insumos também. Esses fatores demostram e justificam a especialização dos gestores que planejam e programam a manutenção, pois essa variação considerada "natural" de um processo manual, pode afetar o orçamento trimestral em $57,43 \%$. Comparando com outras obras já citadas no referencial teórico conclui-se que, a aplicação da modelagem com o software Arena se mostrou eficiente ao fornecer informações relevantes ao planejamento de diferentes áreas em empresas do setor ferroviário, seja relacionado aos suprimentos como no presente estudo, assim como na programação de via permanente nos estudos de Faria (2016) ou no controle de tráfego conforme a pesquisa de Pedreira Junior e Nascimento (2015).

Pesquisas nestas áreas incentivam o investimento na busca por interligações entre a universidade e a iniciativa privada, desta forma cria-se um crescimento competitivo e melhora o serviço logístico ferroviário, que é tão importante para qualquer pais. Vale ressaltar que esta pesquisa não é de caráter conclusivo e está aberta como base para a realização de futuros estudos, porque a literatura recomenda utilizar outras técnicas para realizar previsão de demanda da manutenção. A possível análise de outros fatores, como os ambientais e de satisfação, que também compõem o processo de manutenção dos truques, podem talvez impactar mais o orçamento, que o tempo de execução das tarefas. Pesquisas que abordem mais fatores tem a potencialidade de tornar planejamento de compras mais completo e detalhado, para esse setor. 


\section{REFERÊNCIAS}

Abensur, E. O., Fischmann, A. A., Brunstein, I., \& HO, L. L. (2003). Tendências para o autoatendimento bancário brasileiro: um enfoque estratégico baseado na teoria das filas. RAM. Revista de Administração Mackenzie, 4(2), 40-59.

Alves, A., Dantas, M. J. P., Vale, A., \& Coelho, C. AVALIAÇÃO DE CONFIABILIDADE ATRAVÉS DE SIMULAÇÕES BASEADAS EM TEORIA DE FILAS. In: CONGRESSO NACIONAL DE EXCELÊNCIA EM GESTÃO \& III INOVARSE, 12., 2016, Rio de Janeiro. Anais... . Rio de Janeiro: Cneg \& Inovarse, p. 1 - 19. Disponível em: <http://www.inovarse.org/sites/default/files/T16_151.pdf>. Acesso em: 23 ago. 2018.

de Andrade, E. L. (1998). Introdução à pesquisa operacional: métodos e modelos para a análise de decisão. Livros Técnicos e Científicos. 4., ed. Rio de Janeiro: LTC, 2009.

Rehfeldt, M. J. H. (2015). Estudo da viabilidade dos usos da pesquisa operacional em uma vinícola da Serra Gaúcha. Diálogo, (28), 147-164. Elsevier Brasil.

Camelo, G. R., Coelho, A. S., Borges, R. M., \& de Souza, R. M. (2010). Teoria das filas e da simulação aplicada ao embarque de minério de ferro e manganês no terminal marítimo de ponta da madeira. Cadernos do IME-Série Estatística, 29(2), 1. 2010. Disponível em: https://www.e-publicacoes.uerj.br/index.php/cadest/article/view/15733/11904 Acesso em: 13 nov. 2018

Campos, F. C. D., \& Belhot, R. V. (1994). Gestão de manutenção de frotas de veículos: uma revisão. Gestão \& Produção, 1(2), 171-188. FapUNIFESP (SciELO).

http://dx.doi.org/10.1590/s0104-530x1994000200004. Disponível em:

<http://www.scielo.br/pdf/gp/v1n2/a04v1n2.pdf>. Acesso em: 20 ago. 2018.

Morabito, R., Pureza, V., Fleury, A., Mello, C. H. P., Nakano, D. N., de Lima, E. P., ... \& Sousa, R. (2011). Metodologia de pesquisa em engenharia de produção e gestão de operações. Elsevier Brasil.

dos Santos, J. A. A., \& Grander, G. (2016). Análise e simulação do sistema de estocagem de uma indústria moveleira: um estudo de caso. FaSCi-Tech, 1(6). Disponível em: 
https://www.fatecsaocaetano.edu.br/fascitech/index.php/fascitech/article/view/62 Acesso em: 10 no. 2018.

Duarte, S. L., Pinto, K. C. R., \& Lemes, S. (2009). Integração da Teoria das Filas ao Timedriven ABC Model: uma análise da capacidade ociosa. Enfoque: Reflexão Contábil, 28(1) 40-53. Disponível em: http://periodicos.uem.br/ojs/index.php/Enfoque/article/view/8219 Acesso em: 13 nov. 2018.

De Faria, c. h. f. Análise do planejamento ferroviário: uma abordagem utilizando simulação de eventos discretos (2016). Dissertação de Mestrado. Universidade Federal do Espírito Santó. Disponível em: http://repositorio.ufes.br/bitstream/10/9462/1/tese_10340_Carlos\%20Henrique\%20Fernandes \%20de\%20Faria\%20-\%20\%20Dissertacao_v9.pdf. Acesso em: 20 nov. 2018.

Felix, J. C., Barbosa, L. C. M., \& Valente, A. F. D. A. (2021). Um estudo sobre as condições de trabalho e layout industrial para a realização de mudanças em uma oficina de componentes ferroviários da mrs logística. Exacta. Disponível em: https://periodicos.uninove.br/exacta/article/view/17873/9078. Acesso em: 11 de agosto de 2021.

Fernandes, A. (2012). Simulação de Linha de Produção usando a Plataforma ARENA (Doctoral dissertation, MSc Thesis in Informatics Engineering, Polytechnic Institute of Bragança, Portugal).

Freitas, N. (2015) Fundamentos de vagões MRS. Juiz de Fora: s.n., (Apostila para Treinamento e desenvolvimento de funcionários da MRS Logística.).

GAUDREAULT, J. et al. Distributed operations planning in the softwood lumber supply chain: models and coordination. International Journal of Industrial Engineering: Theory Applications and Practice, v. 17, p. 168-189, 2010.

Hillier, F. S., \& Lieberman, G. J. (2013). Introdução à pesquisa operacional. McGraw Hill Brasil. 
Júnior, J. U. P., \& do Nascimento, A. N. (2016). Representação do transporte de cargas na ferrovia de integração oeste leste com o emprego de simulação e heurística. Simpósio de Pesquisa Operacional e Logística da Marinha-Publicação Online, 2(1), 600-614.São Paulo.

Marcelino, A. R. A., Costa, D. C., Martins, M. H., \& Almeida, M. A. F. (2020). Simulação em 3D de uma linha de embalagem de carretéis MIG/MAG usando o software Simio/3D simulation of a MIG/MAG spool packaging line using Simio software. Brazilian Journal of Business, 2(2), 1328-1342.

Marujo, L. G., Carvalho, D., \& Leitão, M. N. (2010). Otimização de layout utilizando-se o SLP combinado com teoria das filas: Um estudo de caso em uma oficina de rodas e freios de aeronaves. Revista Gestão Industrial, 6(4).

de Medeiros, L. F., Moser, A., \& dos Santos, N. (2015). A simulação computacional como técnica de pesquisa na administração. Revista Intersaberes, 9, 463-485.

Melo, N. A. F., Pessanha, A. M. B., \& Rocha Filho, S. M. D. (2011). ESTUDO DA APLICAÇÃO DO SOFTWARE ARENA EM UM CONTRATO DE PRESTAÇÃO DE SERVIÇO DE MANUTENÇÃO DE INSTRUMENTAÇÃO. Exatas \& Engenharias, 1(02).

MRS LOGÍSTICA(2018). Institucional da Empresa. Disponível em:

<https://www.mrs.com.br> Acesso em: 29 ago.2018

Prado, D. (2009). Teoria das Filas e da Simulação (Vol. 2). Falconi Editora. Ed. Belo Horizonte: editora de Desenvolvimento Gerencial. Serie Pesquisa Operacional, 2009.

Prodanov, C. C., \& de Freitas, E. C. (2013). Metodologia do trabalho científico: métodos e técnicas da pesquisa e do trabalho acadêmico-2 ${ }^{a}$ Edição. Editora Feevale..

Kozechen, A. P., Ferreira, J., de Oliveira, G. D., \& de Fatima Morais, M(2016). EMPREGO DA SIMULAÇÃO COMPUTACIONAL PARA ANÁLISE DO SISTEMA DE FILAS NOS CAIXAS DE UM SUPERMERCADO. Disponível em:

<http://www.abepro.org.br/biblioteca/TN_STP_231_350_28586.pdf>. Acesso em: 28 nov. 2018. 
Pimentel, B. S. (2011). Modelos e algoritmos para planejamento integrado na indústria da mineração. (Tese de Doutorado). UFMG. Belo Horizonte.

Ribas, G., Leiras, A., \& Hamacher, S. (2009). Planejamento de Refinarias Sob Incerteza: Uma Revisão. Anais do XLI SBPO-Simpósio Brasileiro de Pesquisa Operacional, 185-196.

Souza, A. C., Tosoni, L. A., Canassa, R. C., \& de Fátima Morais, M. (2015). Estudo de filas em caixas rápidos de um supermercado utilizando simulação computacional. Revista LatinoAmericana de Inovação e Engenharia de Produção, 3(4), 203-222.

Sfeir, T. D. A. (2016). Planejamento da etapa de aquisição da cadeia de suprimentos da indústria florestal: aplicação em indústria canadense. Dissertação de Mestrado. UFP. Curitiba. Paraná.

Xenos, H. G. (2014). Gerenciando a manutenção produtiva: Melhores práticas para eliminar falhas nos equipamentos e maximizar a produtividade. Falconi Editora. 312 p. 
7. Apêndice A

\begin{tabular}{|c|c|c|c|c|c|}
\hline \multicolumn{7}{|c|}{ Duração do atendimento na etapa de desmontagem } \\
(em horas) \\
\hline 1,307 & 0,391 & 1,467 & 1,584 & 1,317 & 1,46 \\
\hline 0,492 & 1,068 & 1,472 & 1,332 & 0,974 & 1,359 \\
\hline 0,716 & 0,906 & 0,82 & 0,385 & 0,7 & 1,286 \\
\hline 1,147 & 0,732 & 1,123 & 0,641 & 1,332 & 1,214 \\
\hline 1,348 & 1,341 & 1,256 & 0,689 & 0,83 & 0,997 \\
\hline 0,981 & 0,995 & 0,263 & 0,26 & 0,847 & 0,664 \\
\hline 1,12 & 0,9 & 0,998 & 1,066 & 0,423 & 0,883 \\
\hline 0,222 & 0,819 & 0,882 & 0,909 & 0,811 & 1,043 \\
\hline 0,614 & 1,016 & 0,447 & 1,091 & 0,852 & 0,579 \\
\hline 0,866 & 0,691 & 0,395 & 0,94 & 0,752 & 0,583 \\
\hline 0,31 & 0,421 & 0,942 & 0,454 & 0,872 & 0,774 \\
\hline 0,542 & 0,687 & 0,255 & 0,761 & 0,695 & 0,5 \\
\hline 0,468 & 0,749 & 0,68 & 0,725 & 0,387 & 0,248 \\
\hline 0,639 & 0,066 & 1,077 & 0,765 & 0,014 & 1,022 \\
\hline 1,256 & 1,092 & 0,834 & 0,914 & 0,822 & 0,961 \\
\hline 1,176 & 1,168 & 0,649 & 0,448 & 0,694 & \\
\hline Duraçan & d &
\end{tabular}

Duração do atendimento na etapa de recuperação

\begin{tabular}{|c|c|c|c|c|c|}
\hline 0,023 & 0,027 & 0,066 & 0,149 & 0,223 & 0,323 \\
\hline 0,359 & 0,422 & 0,433 & 0,485 & 0,489 & 0,513 \\
\hline 0,556 & 0,582 & 0,585 & 0,603 & 0,623 & 0,63 \\
\hline 0,687 & 0,719 & 0,725 & 0,738 & 0,75 & 0,766 \\
\hline 0,802 & 0,809 & 0,823 & 0,831 & 0,868 & 0,869 \\
\hline 0,913 & 0,946 & 0,962 & 0,986 & 1,009 & 1,017 \\
\hline 1,047 & 1,077 & 1,104 & 1,169 & 1,177 & 1,18 \\
\hline 1,203 & 1,208 & 1,209 & 1,217 & 1,241 & 1,255 \\
\hline 1,26 & 1,284 & 1,294 & 1,303 & 1,314 & 1,316 \\
\hline 1,345 & 1,379 & 1,389 & 1,408 & 1,417 & 1,431 \\
\hline 1,434 & 1,436 & 1,468 & 1,552 & 1,689 & 1,73 \\
\hline 1,731 & 1,749 & 1,752 & 1,794 & 1,803 & 1,858 \\
\hline 1,953 & 1,992 & 1,999 & 2,017 & 2,098 & 2,129 \\
\hline 2,16 & 2,192 & 0,406 & 0,461 & 0,518 & 0,528 \\
\hline 0,584 & 0,655 & 0,682 & 0,689 & 0,705 & 0,746 \\
\hline 0,893 & 0,905 & 1,051 & 1,063 & 1,09 & \\
\hline
\end{tabular}

\begin{tabular}{|c|c|c|c|c|c|}
\hline \multicolumn{7}{|c|}{ Duração do atendimento na etapa de montagem } \\
\hline 2,17 & 3,082 & 1,967 & 1,767 & 1,96 & 1,717 \\
\hline 2,649 & 2,01 & 1,595 & 1,683 & 2,037 & 1,628 \\
\hline 2,228 & 2,012 & 2,095 & 2,512 & 2,177 & 1,584 \\
\hline 1,666 & 2,049 & 1,652 & 2,121 & 1,418 & 1,52 \\
\hline 1,35 & 1,35 & 1,421 & 1,98 & 1,802 & 1,634 \\
\hline 1,606 & 1,559 & 2,275 & 2,254 & 1,644 & 1,819 \\
\hline 1,333 & 1,523 & 1,398 & 1,265 & 1,9 & 1,437 \\
\hline 2,075 & 1,473 & 1,409 & 1,374 & 1,448 & 1,202 \\
\hline 1,626 & 1,2 & 1,759 & 1,106 & 1,334 & 1,605 \\
\hline 1,289 & 1,43 & 1,716 & 1,152 & 1,331 & 1,486 \\
\hline 1,756 & 1,643 & 1,09 & 1,494 & 0,939 & 0,996 \\
\hline 1,227 & 1,064 & 1,493 & 0,945 & 1,002 & 1,142 \\
\hline 1,079 & 0,759 & 0,821 & 0,758 & 1,015 & 1,123 \\
\hline 0,701 & 1,242 & 2,017 & 2,274 & 2,968 & 1,95 \\
\hline 1,66 & 1,753 & 1,984 & 1,897 & 1,973 & 1,793 \\
\hline 1,431 & 1,427 & 1,8 & 1,989 & 1,716 & \\
\hline
\end{tabular}

Fonte: Dados da empresa. 